\title{
A Review of the Rationale for Additional Therapeutic Interventions to Attain Lower LDL-C When Statin Therapy Is Not Enough
}

\author{
Jeffrey G. Shanes
}

Published online: 23 November 2011

(C) The Author(s) 2011. This article is published with open access at Springerlink.com

\begin{abstract}
Statins alone are not always adequate therapy to achieve low-density lipoprotein (LDL) goals in many patients. Many options are available either alone or in combination with statins that makes it possible to reach recommended goals in a safe and tolerable fashion for most patients. Ezetimibe and bile acid sequestrants reduce cholesterol transport to the liver and can be used in combination. Niacin is very effective at lowering LDL, beyond its ability to raise high-density lipoprotein and shift LDL particle size to a less atherogenic type. When statins cannot be tolerated at all, red yeast rice can be used if proper formulations of the product are obtained. Nutrients can also be added to the diet, including plant stanols and sterols, soy protein, almonds, and fiber, either individually or all together as a portfolio diet. A clear understanding of how each of these strategies works is essential for effective results.
\end{abstract}

Keywords Statins · Cholesterol - Bile acid sequestrants · Colesevelam · Cholestyramine - Ezetimibe - Niacin ·

Fibrates - Red yeast rice - Fiber - Plant stanols · Plant sterols . Soy protein $\cdot$ Almonds $\cdot$ Dietary portfolio $\cdot$ LDL cholesterol

J. G. Shanes $(\bowtie)$

Clinical Medicine,

Rosalind Franklin University of Medicine and Science,

Melrose Park, IL, USA

e-mail: jeffsh@ccmconsultants.com

\section{J. G. Shanes}

Consultants in Cardiovascular Medicine,

675 W. North Avenue, Suite 210,

Melrose Park, IL 60160, USA

\section{Introduction}

Statin therapy is the most effective way to reduce lowdensity lipoprotein (LDL) to target goals, and their pleiotropic effects are also thought to contribute additional vasculoprotective benefit. However, often times the LDL goal cannot be achieved with statins alone. This may occur because even at the highest dose of the most effective statin, the ideal LDL is not reached or because, due to financial constraints, the most effective statin may not be affordable. It may also occur because patients have intolerable side effects to what otherwise would be an effective statin dose or for that matter any statin at all. Finally, abnormalities in liver or muscle enzymes may limit their use. These barriers contribute to the fact that only about $60 \%$ of patients at risk are at their LDL target [1]. This article reviews what options are available to help patients reach their LDL goals when statin therapy is not enough.

Physiology of Cholesterol Absorption and Metabolism: Opportunities for Therapy

Cholesterol is an essential structural component of mammalian cell membranes, where it is required to establish proper membrane permeability and is also required for the manufacture of bile acids, steroid hormones, and Vitamin D. Adequate stores of cholesterol are vital and achieved through exogenous sources and endogenous pathways of cholesterol metabolism [2]. Ingestion of cholesterol and its active absorption of cholesterol in the gut and enterohepatic recirculation of bile acids from the gut back to the liver are referred to as exogenous pathways of cholesterol metabolism. Synthesis of cholesterol within the liver and reverse 
transport of cholesterol from the blood back to the liver, either by the direct or indirect pathways, is considered the endogenous component of cholesterol metabolism. The direct pathway utilizes high-density lipoprotein (HDL) receptors, such as scavenger receptor BI (SR-BI), that mediate the selective uptake of cholesterol from HDL. The second mechanism is the indirect one, mediated by cholesterol ester transfer protein (CETP). This protein exchanges triglycerides of very low-density lipoprotein (VLDL) with cholesterol esters of HDL. The VLDL is then processed to LDL and removed from the circulation by the LDL receptor pathway. The triglycerides are not stable in HDL but degraded by hepatic lipase, converting them to small HDL particles where they are capable of picking up cholesterol from cells by interaction with the ATP-binding cassette transporter A1 (ABCA1) and then unloading their cholesterol content back to the liver by the direct pathway. Because cholesterol is not water soluble, its transport through the blood requires it to be carried by watersoluble lipoproteins. The type of lipoprotein also determines how the triglycerides and cholesterol within them are metabolized, and to what organs and tissues in the body they are delivered. Most notably, apolipoprotein B (apoB) is the primary apolipoprotein of LDL. It is responsible for delivering cholesterol to tissues and acts as a ligand for LDL receptors in various cells throughout the body, thus allowing the influx of cholesterol into the cells. ApoA-I, on the other hand, is the major protein component of HDL. This protein has the exact opposite effect of ApoB and promotes cholesterol efflux from tissues and reverse transport to the liver for excretion [3].

Thus, we see that there are numerous targets for therapy that can be utilized to reduce LDL by affecting either the exogenous or endogenous pathways of cholesterol metabolism or both simultaneously. These include a reduction of cholesterol in the diet along with medications or nutrients that reduce the absorption of ingested cholesterol within the gut or that prevent entero-hepatic recirculation of bile acids. All these methods will reduce the total cholesterol pool by affecting the exogenous pathways of cholesterol metabolism. They also work extremely well when used in combination with strategies that block the endogenous pathways of cholesterol metabolism, such as statins. When the cholesterol pool is depleted, the liver can replenish its supply of cholesterol in two ways. First it can do so by increasing the activity of HMG-CoA reductase, the ratecontrolling enzyme for the production of cholesterol, and secondly by absorbing more cholesterol from the blood by upregulation of LDL receptors. However, if cholesterol synthesis is blocked by statins then the only way for the liver to obtain more cholesterol is by further upregulation of LDL receptors, causing even greater clearance of LDL from the blood.
This article summarizes how each step of the process of cholesterol metabolism can be altered in order to achieve the LDL goal when statins are not enough.

\section{Strategies That Affect Exogenous Cholesterol Metabolism}

Reduction of Fat and Cholesterol in the Diet

It is an accepted principle that a key component of lowering cholesterol in the blood is diet. Certainly maintaining an ideal weight and regular exercise is essential to help prevent the metabolic syndrome and reduce the likelihood of developing diabetes. However, the effect on total and LDL cholesterol by limiting dietary intake of cholesterol and fat in the diet appears to be relatively small. In a study by Hunninghake et al. [4] in 1993, 97 patients underwent consecutive 9-week periods of two different dietary regimens. Each dietary regimen also included an additional 9 weeks of either placebo or $20 \mathrm{mg}$ of lovastatin. The dietary regimens were either a diet high in fat and cholesterol intended to resemble the average US diet or a National Cholesterol Education Program (NCEP) step 2 diet. The high fat and cholesterol diet provided approximately $40 \%$ of calories as fat, with $15 \%$ saturated, $14 \%$ monounsaturated, and $8 \%$ polyunsaturated fatty acids, and a cholesterol intake of 350 to $400 \mathrm{mg}$ per day. The NCEP step 2 diet consisted of very low levels of saturated fat $(<7 \%)$ and cholesterol $(<200 \mathrm{mg})$. The reduction in the total cholesterol and LDL between the two diets was only $5 \%$ and apoB only decreased by $2 \%$. For comparison, lovastatin in the highfat diet decreased LDL by $27 \%$ and in the low-fat diet plus lovastatin, a further 5\%. In another study, 120 patients were randomized to receive either their "habitual diet" or a Mediterranean diet for 12 weeks [5]. During those 12 weeks, each group was further randomized to receive $20 \mathrm{mg}$ of simvastatin or placebo. After 12 weeks, the simvastatin groups were crossed over to receive placebo for 12 weeks and the placebo groups crossed over to receive simvastatin for 12 weeks. The Mediterranean diet consisted of no more than $10 \%$ energy from saturated fats and trans-unsaturated fats, and the cholesterol intake was less than $250 \mathrm{mg}$ per day. It also included $4 \mathrm{~g}$ per day of omega-3 fatty acid of plant and marine origin. This diet also required increased intake of fruit, vegetables, and soluble fiber. The effect of diet alone on reducing LDL was about $11 \%$, simvastatin about $30 \%$, and the combination of both $40 \%$. Thus, even in the patients who are dietary compliant, reductions of LDL cholesterol may only be in the $5 \%$ to $10 \%$ range and usually other strategies to reach the LDL goal will be required. 


\section{Bile Acid Sequestrants}

Bile acid sequestrants (BAS) work by blocking one of the exogenous pathways of cholesterol metabolism, namely enterohepatic recirculation. About $1,000 \mathrm{mg}$ of cholesterol daily is secreted into bile and subsequently into the intestine. These bile acids are essential to emulsify and solubilize lipids for intestinal absorption. About $95 \%$ of the bile acids secreted into the intestines are reabsorbed through enterohepatic recirculation. Bile acid sequestrants bind intestinal bile acids in the gut, forming insoluble complexes that are then excreted. By blocking enterohepatic recirculation and depleting the liver of ready-made bile acids, the liver increases synthesis of cholesterol and up-regulates LDL receptors on its surface, which results in increased clearance of LDL cholesterol from the systemic circulation [6].

Earlier generations of BAS, such as cholestyramine, have been demonstrated to be effective in lowering total and LDL cholesterol as well as reducing mortality and cardiovascular events ( $7 \%$ and $24 \%$, respectively). However, they have not been generally well tolerated due to significant gastrointestinal (GI) side effects, including abdominal pain, heartburn, bloating, and constipation [7].

A new generation of BAS is currently available and is more effective and better tolerated than cholestyramine [6]. Colesevelam is a high-capacity bile acid-binding agent and has been reported to be four to six times more potent than older BAS and reduces LDL cholesterol in a dosedependent manner. In a double-blind study, colesevelam, at a dose of $4.5 \mathrm{~g} / \mathrm{d}$, reduced median LDL cholesterol by $20 \%$, raised HDL cholesterol by $3 \%$ to $4 \%$ and nonsignificantly raised triglycerides by $5 \%$ to $10 \%$ [8]. This compares with a reduction in LDL in the Lipid Research Clinics Coronary Primary Prevention Trial (LRC) with cholestyramine of only $12.6 \%$ versus placebo. The response is rapid, and maximal reductions in LDL occur within 2 two weeks of treatment. In addition, although GI side effects do occur, they are milder and occur less often than with cholestyramine. This is because colesevelam is a water-absorbing gel that creates a soft gelatinous material that may explain its better-tolerated GI side-effect profile. The overall incidence of GI side effects as compared to placebo was $4 \%$ for constipation, $5 \%$ for dyspepsia, and $0.3 \%$ for nausea. Overall, discontinuation from the drug because of adverse GI side effects as compared to placebo was about $2 \%$. Because all BAS are not absorbed, systemic side effects do not occur. However, because colesevelam may affect absorption of certain drugs, it should not be administered within $4 \mathrm{~h}$ of taking glyburide, levothyroxine, and oral contraceptives containing ethinyl estradiol and norethindrone and phenytoin. Colesevelam is also indicated for the treatment of type 2 diabetes and reduces HBA1c by about $0.5 \%$ and fasting blood sugars by about $14 \%$. Therefore, it is an ideal drug for diabetic patients who require add-on therapy for getting LDL to goal. The mechanism for this improvement is not well understood but has been reviewed [9].

\section{Combination Therapy with Colesevelam and Statins}

As described, when BAS are given, the liver repletes its stores of cholesterol by increasing synthesis and upregulating LDL receptors. However, if statins are given in combination with BAS, increased cholesterol synthesis cannot occur and only up-regulation of LDL receptors is available to the liver to increase its stores of cholesterol. In that situation, the cholesterol-lowering effect of each drug is magnified. In a pooled analysis of three trials that analyzed the effects of adding colesevelam to simvastatin, pravastatin, or atorvastatin, the additional LDL lowering was $9.2 \%$ [10]. Unfortunately, no outcome data are available to compare high-dose statins and their effect on reducing cardiovascular events versus low-dose statins plus colesevelam. Because the beneficial effects of statins are thought to be partly related to their pleiotropic effects, it is possible that equal reduction in cholesterol by combination therapy with low-dose statin plus colesevelam versus high doses of statins alone might not translate into similar reduction in cardiovascular morbidity. It is noteworthy, however, that in the pooled data described above, further lowering of the cholesterol with the addition of colesevelam to statins did result in further lowering of CRP levels, which is a marker of future cardiovascular events. There are also no studies that compare overall tolerability of high-dose statins with low-dose statins plus colesevelam, but in practice, when myalgias increase creatine kinase or abnormal liver function tests preclude giving higher doses of statins, this approach usually works well. There are also no studies that have systematically looked at the addition of colesevelam to high doses of statin in terms of tolerability, outcome data, or quantified the additional LDL lowering that could be expected. In practice, however, any type of combination therapy that achieves recommended LDL goals in a way that is tolerable, achieves long-term compliance, and can be monitored for safety seems reasonable and practical.

\section{Ezetimibe}

Ezetimibe, like BAS, blocks the exogenous pathway of cholesterol metabolism and lowers LDL to a similar degree as colesevelam. Ezetimibe, in a dose of $10 \mathrm{mg}$, lowers LDL by about $18 \%$ [11] and recent studies have shown similar results with $5 \mathrm{mg}$ compared with $10 \mathrm{mg}$ in a double-blinded study [12]. However, BAS reduces cholesterol available to the liver by preventing enterohepatic absorption of the 
cholesterol found in the bile and is not absorbed. Ezetimibe is rapidly absorbed, with peak concentrations occurring within 1 to $2 \mathrm{~h}$ post-administration and extensively metabolized in the liver $(>80 \%)$ to the pharmacologically active ezetimibe glucuronide followed by enterohepatic recycling and slow elimination. Ezetimibe glucuronide is then excreted into the gut, localizes at the brush border of the small intestine, and inhibits the absorption of ingested cholesterol by about 50\% [13]. Because ezetimibe is systemically absorbed, this difference has implications as to the safety of administering the drug during pregnancy, to children, and to nursing mothers, as opposed to BAS, which are not absorbed systemically.

Because ezetimibe and bile acid sequestrants work by reducing intestinal absorption of cholesterol by different mechanisms, their effects are additive and in several studies the combination of the two were safe and further reduced LDL by an additional $10 \%$ to $20 \%$ compared to either drug alone [14]. Similarly, combining ezetimibe with statins further reduces LDL. In one study, a strategy of doubling the dose of rosuvastatin was compared to adding $10 \mathrm{mg}$ of ezetimibe to the same statin dose. When ezetimibe was added to either 5 or $10 \mathrm{mg}$ of rosuvastatin, there was about a $21 \%$ further reduction in LDL as opposed to only a $6 \%$ further reduction when the statin dose was doubled [15].

One study evaluated the effects of adding colesevelam to patients with familial hypercholesterolemia already on high-dose statins plus ezetimibe and found that the addition of colesevelam reduced the LDL by another $18 \%(-11.8 \%$ to $-25 \%$ ) with no safety issues [16]. Thus, the combination of a potent statin, ezetimibe, and colesevelam has the potential to decrease the LDL by as much as $75 \%$ to $80 \%$ from baseline depending on the statin used. As with all studies evaluating BAS, GI side effects were more common in the colesevelam group as compared to placebo.

It would appear, however, based on the ARBITER 6HALTS trial, that patients whose LDL is below $100 \mathrm{mg} /$ $\mathrm{dL}$ and require the addition of a second drug to reach their LDL goal should receive niacin rather than ezetimibe. In that trial, patients with $\mathrm{CAD}$ or CAD equivalents already on statins whose LDL was below $100 \mathrm{mg} / \mathrm{dL}$ were randomized to receive either niacin extended-release (ER) $2,000 \mathrm{mg} / \mathrm{d}$ or ezetimibe $10 \mathrm{mg} / \mathrm{d}$ [17]. The baseline LDL in each group was about $80 \mathrm{mg} / \mathrm{dL}$ and the on-treatment LDL was about $66 \mathrm{mg} / \mathrm{dL}$ in the ezetimibe group and about $71 \mathrm{mg} / \mathrm{dL}$ in the niacin-treated group. However, only the niacin group demonstrated regression of vascular disease as determined by measurement of carotid intima-media thickness (CIMT) [17].

In clinical practice, however, the LDL levels that need to be treated are usually much higher than those in the ARBITER 6-HALTS trial and every study that has been done with any method to lower LDL, such as statins [18],
BAS [7], and illeal bypass [19] have all shown a log-linear relationship between LDL lowering and a reduction in $\mathrm{CV}$ events regardless of the method used. There seems nothing unusual about ezetimibe that would suggest that lowering LDL with it in patients who cannot otherwise achieve their LDL goal with statins would not benefit. In fact, a reduction in C-reactive protein (CRP) levels, a marker of risk, has been noted with ezetimibe alone and when added to statin therapy, just as they have been reduced with BAS [20] as well as with statins. In summary, within the usual range of LDL levels being treated, ezetimibe is a very reasonable option.

\section{Strategies That Affect Endogenous Cholesterol Metabolism}

Niacin

Niacin is the best agent to raise HDL cholesterol. It also increases LDL particle size and lowers lipoprotein(a) (Lp (a)), all of which are thought to have vasculoprotective effects [21]. However, niacin is also effective in lowering total and LDL cholesterol. The effect appears to be dose responsive and lowers total cholesterol by about $5 \%$ $(1,000 \mathrm{mg}), 10 \%(2,000 \mathrm{mg})$, and $16 \%(3,000 \mathrm{mg})$ and LDL $9 \%(1,000 \mathrm{mg}), 17 \%(2,000 \mathrm{mg})$, and $21 \%(300 \mathrm{mg})$ [22]. The combination of niacin with statins is additive. In one study, $40 \mathrm{mg}$ of lovastatin decreased LDL by $32 \%$, niacin ER at a dose of 2,000 $\mathrm{mg}$ reduced LDL by $14 \%$, and the combination decreased LDL by $42 \%$ [23].

Niacin appears to reduce total and LDL cholesterol by several mechanisms [24]. First, the liver does not directly produce LDL. It does produce VLDL, which is then metabolized to intermediate-density lipoproteins (IDL) and then into LDL. Niacin reduces the quantity of VLDL produced in the liver in the following way. Two of the major components of VLDL are triglycerides (TG) and apoB, which are both produced in the liver. The initial substrates for TG synthesis are glycerol and free fatty acids. Niacin produces a large and rapid decrease in the release of free fatty acids from adipose tissue, thus making them unavailable to produce triglycerides in the liver. In addition, niacin also directly inhibits the hepatocyte microsomal diacylglycerol acyltransferase-2 (DGAT2), which is the key enzyme that catalyzes the transformation of diacyl glycerol to triglycerides. When TG synthesis is reduced, less is available to lipidate apoB, which in turn results in intrahepatic degradation of apoB as opposed to secretion. The overall result of all this is to make less VLDL available to produce LDL. Secondly, besides decreasing the quantity of VLDL produced, niacin also decreases the size of the VLDL particle. When triglyceride levels are high, large, 
triglyceride-rich VLDL particles are produced and cholesterol-ester transfer protein (CETP) exchanges TG in the largest VLDL particles for cholesterol esters in the large LDL particles. The acquired excess triglycerides in the large LDL particles undergo rapid hydrolysis by hepatic lipase, which leads to the formation of small dense LDL particles, which have less affinity for hepatic LDL receptors, which prolongs their presence in the circulation. Thus, overall reduced production of LDL and increased clearance of LDL by niacin reduces LDL and total cholesterol levels.

\section{Fibrates}

The action of fibrates on the lipid profile is similar to niacin in many ways, including a reduction in triglyceride levels, an increase in HDL levels, and a shift of the LDL particle size to a larger, less atherogenic type. However, they do so by a different mechanism of action than niacin [25]. They activate PPAR $\alpha$ nuclear hormone receptors, leading to the induction of lipoprotein lipase (LPL) expression and a repression of the apoC-III gene at the transcriptional level. This leads to increased oxidative metabolism of free fatty acids in the liver, thus leading to decreased VLDL production. There is also enhanced LPL-mediated catabolism of VLDL because apoC-III on the VLDL particle makes them less susceptible to the action of LPL. It is this difference in mechanism of action that probably makes their LDL lowering effect much less than niacin. Most studies have seen only a $5 \%$ reduction in LDL, with no additive benefit to statins, and a paradoxical increase in LDL in patients with high baseline triglyceride levels of up to $14 \%$ to $45 \%[26,27]$. Therefore, fibrates as an agent to specifically lower total cholesterol and LDL appear to have limited use, although their other benefits are important.

\section{Red Yeast Rice}

Red yeast rice has been used as a Chinese cuisine and medicinal agent since the Ming dynasty of 1368 to 1644 . It contains isoflavanes that have antioxidant properties, sterols, a group of compounds that are known to block cholesterol absorption in the gut, and polyketides [28]. Polyketides are secondary metabolites from bacteria, fungi, plants, and animals. Macrolide antibiotics, amphotericin, and tetracycline are all derived from polyketides [29]. Monacolin K, which is lovastatin, is a polyketide found in red yeast rice along with eight other monoclonins, all of which probably reduce cholesterol synthesis in the liver. They do so by reversible competitive inhibition of HMGCoA reductase, the enzyme that catalyzes the conversion of HMG-CoA to mevalonate. Mevalonate is a required building block for cholesterol biosynthesis. Isolated mon- acolin $\mathrm{K}$, lovastatin, and synthetically produced statins are widely accepted as the agent of choice to reduce serum cholesterol levels because of their predictability, safety, and outcome data. Although myalgias are a significant obstacle to using them, many options have been suggested that may increase their tolerability. These include supplementation with vitamin D [30], coenzyme Q10 [31], use of small doses of rosuvastatin given twice weekly [32], or the use of fluvastatin in the XL formulation [33]. However, there are significant numbers of patients that cannot take any dose of a statin because of myalgias. Some data suggest that when any dose of a statin cannot be tolerated, red yeast rice may be an alternative. In a study by Heber et al. [34], 83 patients on a step 1 AHA diet were randomized to receive placebo or $2.4 \mathrm{~g}$ of red yeast rice daily and followed for 12 weeks. Compared with placebo, total cholesterol was reduced by about $18 \%$ and LDL by $23 \%$. There was no liver toxicity or myalgia in either group [34]. In a study by Becker et al. [35], 62 patients with a history of discontinuation of statins due to myalgias were randomized to receive $1,800 \mathrm{mg}$ of red yeast rice or placebo for 12 weeks. The mean reduction in LDL at 24 weeks was $21 \%$ and for TC was $16 \%$. One patient in the placebo group and two in the red yeast rice group developed intolerable myalgias, none of whom had an increase in CK levels, and no patients developed evidence of liver toxicity [35]. The amount of lovastatin in the red yeast rice group in both studies was only 5 to $7 \mathrm{mg}$ and cannot explain the significant drop in cholesterol. It is hypothesized that it is the combination of all the monaclonins and other compounds in the red yeast rice that may explain the significant cholesterol reductions seen. Although these trials are relatively small, in 2006 Liu et al. [36] performed a meta-analysis of 93 double-blind randomized trials using red yeast rice and found similar degrees of cholesterol lowering. To determine if red yeast rice can reduce cardiovascular events, a large, double-blind, randomized prospective study was performed in 5,000 patients post-myocardial infarction (MI) who were randomized to receive red yeast rice or placebo and followed for an average of 4.5 years. Total cholesterol levels declined by $13 \%$ from baseline and LDL by $20 \%$. Adverse effects were reported to be similar to placebo, but specific details were not given. The reduction in total $\mathrm{CV}$ events (nonfatal MI or a cardiac death from any cause) was striking, with a $45 \%$ reduction in major coronary events, $62 \%$ reduction in the occurrence of nonfatal MI, and 32\% reduction in fatal coronary events [37].

A significant problem with recommending over-thecounter red yeast rice products to patients is that there is no standardization among marketed products. In one study that analyzed the amount of monaclonin in 12 commercially available products, there was marked variability, with total monaclonins varying from 0.31 to $11.15 \mathrm{mg} /$ capsule, 
although each capsule was labeled as "600 mg/capsule" [38]. Because of this, we have chosen to make available to our patients the two RYR compounds that are commercially available and used in the Heber and Becker studies, both of which were analyzed for monaclonin content and were found to be effective. We have obtained very consistent results by doing so and they have been well tolerated.

In summary, we believe that red yeast rice should be used only as a last resort when statins can absolutely not be tolerated. However, in those circumstances, there are much data to support its use if commercially available regimens that have been well analyzed are made available to patients.

\section{Adding Nutrients and Fiber to the Diet}

Dietary fiber is a collective term for a variety of plant substances that are resistant to digestion in humans. There are two types: water soluble and insoluble. Water-soluble fiber, such as oats, psyllium, pectin, and guar gum, have been shown to cause some reduction in total and LDL cholesterol, but the degree to which they lower cholesterol has been quite varied. Mechanisms of action proposed for this effect have included a bile acid binding effect similar to bile acid sequestrants, a dietary cholesterol binding effect, inhibition of hepatic fatty acid synthesis byproducts of fermentation, and increased satiety leading to decreased caloric intake. In a meta-analysis of 67 controlled trials by Brown et al. [39], it was concluded that all the various soluble fibers reduce total cholesterol and LDL by similar and small amounts within the practical range of possible intake, the dose response being about a $5-\mathrm{mg} / \mathrm{dL}$ reduction in total and LDL cholesterol for $3 \mathrm{~g}$ of soluble fiber. This would be the equivalent of 1.5 servings of a commercially available fiber supplement or three servings of oatmeal per day.

\section{Plant Stanols and Sterols}

Plant stanols added to the diet have consistently shown a reduction in LDL cholesterol. Because phytosterols are structurally similar to cholesterol, they compete with cholesterol for absorption in the digestive system when they are consumed. A meta-analysis of 41 trials showed that the intake of $2 \mathrm{~g}$ of stanols or sterols reduced LDL by about $10 \%$ but higher doses added little. They do not affect the absorption of fat-soluble vitamins $\mathrm{A}, \mathrm{D}, \mathrm{E}$, and $\mathrm{K}$ [40]. The effects are additive to statins but are not additive to bile acid sequestrants nor to ezetimibe [41, 42]. They are commercially available in many forms that should suit most patients' preferences. These include chewable tablets $(0.7 \mathrm{~g}$ per tablet) orange juice, spreads, cheeses, soymilk, rice milk, and breads (all fortified with plant sterols or stanols). A search on the internet using "dietary supplements with plant stanols" will be quite useful for finding all of these.
Soy Protein

Adding soy protein to the diet appears to result in some reduction in total and LDL cholesterol. In a meta-analysis of studies that substituted soy protein for animal protein, the LDL-lowering effect ranged from $7 \%$ in the quartile with the lowest cholesterol $(127-197 \mathrm{mg} / \mathrm{dL})$ and up to $24 \%$ in the group with the highest cholesterol $(>355 \mathrm{mg} /$ dL) [43]. Studies have also shown that by simply adding soy protein to patients' regular diets can lower LDL levels by $4 \%$ and in patients with the highest baseline levels by $8 \%$ [44]. Much has been debated as to the benefit of alcohol-extracted soy protein, which is devoid of isoflavones, versus non-alcohol-extracted soy protein, but the results are mixed. Overall, it appears that the combination of soy protein with isoflavanes works best and this is the recommendation of the American Heart Association, which suggests that the addition of 25 to $50 \mathrm{~g}$ of soy protein will decrease the LDL from $4 \%$ to $8 \%$ depending on the baseline LDL level. No clear explanation as to the mechanism of action of soy protein has emerged, though many theories have been proposed [45]. A search on the internet for sources of soy protein is very helpful. As an example, one soy burger with a cup of soy milk and onehalf cup of edamame will yield about $30 \mathrm{~g}$ of soy protein.

\section{Almonds}

Almonds and other nuts have been demonstrated to significantly reduce LDL in a dose-dependent manner, and a number of epidemiologic trials have demonstrated a reduction in cardiovascular risk. Their benefits may be related to their high content of polyunsaturated/saturated fatty acids (PUFA/SFA), nut protein, plant sterols, fiber, and in addition they are the best natural source of vitamin $\mathrm{E}$ and arginine, a precursor of nitric oxide [46]. In a small, randomized crossover study, 27 men and women consumed three isoenergetic diets. One group ate no almonds; one group $36 \mathrm{~g} / \mathrm{d}$ of almonds, and the third group $73 \mathrm{~g} / \mathrm{d}$ of almonds (for reference, one-quarter cup of almonds equals about $70 \mathrm{~g}$, which contains about $205 \mathrm{cal}$ ). This resulted in a $4.4 \%$ and $9.4 \%$ reduction in LDL for the low-dose and high-dose almond groups compared to the non-almond consuming group. In the group that received no almonds or half almonds, muffins were substituted to provide equal amounts of fiber, protein, and SFA and PUFA as in the almond groups [47].

\section{Dietary Portfolio}

As we have seen, plant stanols, dietary fiber, soy protein, and almonds all cause mild reductions in cholesterol by different mechanisms. A low-fat, low-cholesterol diet, with 
the addition of all of these nutrients, is referred to as a portfolio diet. In one study, hypercholesterolmic patients were randomized to receive either a diet very low in saturated fats (controls), the same diet plus lovastatin $20 \mathrm{mg}$, or a portfolio diet high in viscous fiber $(9.8 \mathrm{~g} /$ $1,000 \mathrm{kcal})$, plant stanols $(1 \mathrm{~g} / 1,000 \mathrm{kcal})$, soy protein $(21.4 \mathrm{~g} / 1,000 \mathrm{kcal})$, and almonds $(14 \mathrm{~g} / 1,000 \mathrm{kcal})$. The LDL reduction in the control group was $8 \%$, in the lovastatin group $30.9 \%$, and in the portfolio diet group $28.6 \%$. The difference in LDL cholesterol between the portfolio diet group and lovastatin group was not statistically significant. In addition, only a minimal fall in CRP of $10 \%$ was seen in the control group, but in the portfolio group and the lovastatin group the reduction was about $30 \%$, with no statistically significant difference between the two groups [48].

\section{Conclusions}

Many patients who are at risk for coronary disease present treatment challenges with regards to achieving their LDL goal. This article reviewed numerous proven and well tolerated options that are available to help each patient reach their LDL goal and help prevent future cardiovascular events. It also reviewed the mechanism of each and demonstrated which combinations are effective and rational, thus allowing physicians to reach success at achieving LDL goals in a wide group of patients.

Acknowledgments Special thanks to Vilma Rodriguez, Diane Paulini, and Melissa Rosen in the preparation of this manuscript.

Disclosure J.G. Shanes has received honoraria for speaking for AstraZeneca and Abbott, has had travel expenses covered by AstraZeneca, and has participated in clinical trials in conjunction with AstraZeneca, Merck, GlaxoSmithKline, and Hoffman La Roche.

Open Access This article is distributed under the terms of the Creative Commons Attribution Noncommercial License which permits any noncommercial use, distribution, and reproduction in any medium, provided the original author(s) and source are credited.

\section{References}

1. Pearson TA, Laurora I, Chu H, Kafonek S. The lipid treatment assessment project. Arch Intern Med. 2000;160:459-67.

2. Russell DW. Cholesterol biosynthesis and metabolism. Cardiovasc Drugs Ther. 1992;6:103-10.

3. Hill SA, McQueen MJ. Reverse cholesterol transport-a review of the process and its clinical implications. Clin Biochem. 1997;30:517-25.

4. Hunninghake DB, Stein EA, Dujovne CA, et al. The efficacy of intensive dietary therapy alone or combined with lovastatin in outpatients with hypercholesterolemia. N Engl J Med. 1993;328:1213-9.

5. Jula A, Marniemi J, Risto H, et al. Effects of diet and simvastatin on serum lipids, insulin, and antioxidants in hypercholesterolemic men: a randomized controlled trial. JAMA. 2002;287:598-605.

6. Robinson DM, Keating GM. Colesevelam: a review of its use in hypercholesterolemia. Am J Cardiovasc Drugs. 2007;6:453-65.

7. The lipid research clinics program: the lipid research clinics coronary primary prevention trial results: I. Reduction in incidence of coronary heart disease. JAMA 1984;251:351-64.

8. Insull W, Toth P, Mullican W, et al. Effectiveness of colesevelam hydrochloride in decreasing LDL cholesterol in patients with primary hypercholesterolemia: a 24-week randomized controlled trial. Mayo Clin Proc. 2001;76:971-82.

9. Bays HE, Goldberg RB, Truitt KE, Jones MR. Colesevelam hydrochloride therapy in patients with type 2 diabetes mellitus treated with metformin. Arch Intern Med. 2008;168:1975-83.

10. Bays HE, Davidson M, Jones MR, Abby SL. Effects of colesevelam hydrochloride on low-density lipoprotein cholesterol and highsensitivity C-Reactive protein when added to statins in patients with hypercholesterolemia. Am J Cardiol. 2006;97:1198-205.

11. Pandor A, Ara RM, Tumor I, et al. Ezetimibe monotherapy for cholesterol lowering in 2,722 people: systematic review and metaanalysis of randomized controlled trials. J Int Med. 2008;265:56880.

12. Baruch L, Agarwal S, Gupta B, et al. Effect on serum lipid levels of switching dose of ezetimibe from 10 to $5 \mathrm{mg}$. Am J Cardiol. 2009;103:1568-71.

13. Kosoglou T, Statkevich P, Johnson-Levonas AO, et al. Ezetimibe a review of its metabolism, pharmacokinetics and drug interactions. Clin Pharmacokinet. 2005;44:467-94.

14. Zema MJ. Colesevelam HCI and ezetimibe combination therapy provides effective lipid-lowering in difficult-to-treat patients with hypercholesterolemia. Am J Ther. 2005;12:306-10.

15. Bays HE, Davidson MH, Massaad R, et al. Safety and efficacy of ezetimibe added on to rosuvastatin 5 or $10 \mathrm{mg}$ versus up-titration of rosuvastatin in patients with hypercholesterolemia (the ACTE study). Am J Cardiol. 2011;108:523-30.

16. Huijgen R, Abbink EJ, Bruckert E, et al. Colesevelam added to combination therapy with a statin and ezetimibe in patients with familial hypercholesterolemia: a 12-week, multicenter, randomized, double-blind, controlled trial. Clin Ther. 2010;32:615-25.

17. Taylor AJ, Villines TC, Stanek EJ, et al. Extended-release niacin or ezetimibe and carotid intima-media thickness. N Engl J Med. 2009;361:2113-22.

18. Grundy SM, Cleeman JI, Merz NB, et al. Implications of recent clinical trials for the national cholesterol education program adult treatment Panel III guidelines. Circulation. 2004;110:227-39.

19. Buchwald H, Varco RL, Boen JR, et al. Effective lipid modification by partial ileal bypass reduced long-term coronary heart disease mortality and morbidity: five-year posttrial followup report from the POSCH. Arch Intern Med. 1998;158:1253-61.

20. Pearson TA, Ballantyne CM, Veltri E, et al. Pooled analyses of effects on C-Reactive protein and low density lipoprotein cholesterol in placebo-controlled trials of ezetimibe monotherapy or ezetimibe added to baseline statin therapy. Am J Cardiol. 2009;103:369-74.

21. Morgan JM, Carey CM, Lincoff A, Capuzzi DM. The effects of niacin on lipoprotein subclass distribution. Prev Cardiol. 2004;7:182-9.

22. Goldberg A, Alagona Jr P, Capuzzi DM, et al. Multiple-dose efficacy and safety of an extended-release form of niacin in the management of hyperlipidemia. Am J Cardiol. 2000;85:1100-5.

23. Ballantyne CM, Davidson MH, McKenney JM, et al. Comparison of the efficacy and safety of a combination tablet of niacin extended-release and simvastatin with simvastatin $80 \mathrm{mg}$ mono- 
therapy: the SEACOAST II (high dose) study. J Clin Lipidol. 2008;2:79-90.

24. Kamanna VS, Kashyap ML. Mechanism of action of niacin on lipoprotein metabolism. Curr Atheroscler Rep. 2000;2:36-46.

25. Staels B, Dallongeville J, Auwerx J, et al. Mechanism of action of fibrates on lipid and lipoprotein metabolism. Circulation. 1998;98:2088-93.

26. Jones PH, Davidson MH, Goldberg AC, et al. Efficacy and safety of fenofibric acid in combination with a statin in patients with mixed dyslipidemia: pooled analysis of three phase 3, 12-week randomized, controlled studies. J Clin Lipidol. 2009;3:125-37.

27. Goldberg AC, Schonfeld G, Feldman EB, et al. Fenofibrate for the treatment of type IV and V hyperlipoproteinemias: a double-blind, placebo-controlled multicenter US study. Clin Ther. 1989;1:69-83.

28. Wang J, Lu Z, Chi J. Current therapeutic research, clinical and experimental. Curr Ther Res. 1997;58:964-78.

29. Staunton J, Weissman KJ. Polyketide biosynthesis: a millennium review. R Soc Chem. 2001;18:380-416.

30. Ahmed W, Khan N, Glueck CJ, et al. Low serum $25(\mathrm{OH})$ vitamin D levels $(<32 \mathrm{ng} / \mathrm{mL})$ are associated with reversible myositismyalgia in statin-treated patients. Transl Res. 2009;153:11-6.

31. Marcoff L, Thompson PD. The role of coenzyme Q10 in statinassociated myopathy a systematic review. J Am Coll Cardiol. 2007;49:2231-7.

32. Gadarla M, Kearns AK, Thompson PD. Efficacy of rosuvastatin $(5 \mathrm{mg}$ and $10 \mathrm{mg}$ ) twice a week in patients intolerant to daily statins. Am J Cardiol. 2008;101:1747-8.

33. Bruckert E, Hayem G, Dejager S, et al. Mild to moderate muscular symptoms with high-dosage statin therapy in hyperlipidemic patients-the PRIMO study. Cardiovasc Drugs Ther. 2005;19:403-14.

34. Heber D, Yip I, Ashley JM, et al. Cholesterol-lowering effects of a proprietary Chinese red-yeast-rice dietary supplement. Am J Clin Nutr. 1999;69:231-6.

35. Becker DJ, Gordon RY, Halbert SC, et al. Red yeast rice for dyslipidemia in statin-intolerant patients a randomized trial. Ann Intern Med. 2009;150:830-9.

36. Liu J, Zhang J, Grimsgaard S, et al. Chinese red yeast rice (Monascus purpureus) for primary hyperlipidemia: a metaanalysis of randomized controlled trials. Chin Med. 2006;1:4.
37. Lu Z, Kou W, Du B, et al. Effect of Xuezhikang, an extract from red yeast chinese rice, on coronary events in a Chinese population with previous myocardial infarction. Am J Cardiol. 2008;101:1689-93.

38. Gordon RY, Cooperman T, Obermeyer W, Becker DJ. Marked variability of monacolin levels in commercial red yeast rice products. Arch Intern Med. 2010;170:1722-7.

39. Brown L, Rosner B, Willett WW, Sacks FM. Cholesterol-lowering effects of dietary fiber: a meta-analysis. Am J Clin Nutr. 1999;69:30-42.

40. Katan MB, Grundy SM, Jones P, et al. Efficacy and safety of plant stanols and sterols in the management of blood cholesterol levels. Mayo Clin Proc. 2003;78:965-78.

41. Jakulj L, Trip MD, Sudhop T, et al. Inhibition of cholesterol absorption by the combination of dietary plant sterols and ezetimibe: effects on plasma lipid levels. J Lipid Res. 2005;46:2692-8.

42. Linnebur SA, Capell WH, Saseen JJ, et al. Plant sterols added to combination statin and colesevelam hydrochloride therapy failed to lower low-density lipoprotein cholesterol concentrations. J Clin Lipidol. 2007;1:626-33.

43. Anderson JW, Johnstone BM, Cook-Newell ME. Meta-analysis of the effects of soy protein intake on serum lipids. N Engl J Med. 1995;333:276-82.

44. Crouse III JR, Morgan T, Terry JG, et al. A randomized trial comparing the effect of casein with that of soy protein containing varying amounts of isoflavones on plasma concentrations of lipids and lipoproteins. Arch Intern Med. 1999;159:2070-6.

45. Erdman Jr JW. Soy protein and cardiovascular disease: a statement for healthcare professionals from the nutrition committee of the AHA. Circulation. 2000;102:2555-9.

46. Fraser GE. Nut consumption, lipids, and risk of a coronary event. Clin Cardiol. 1999;22:III-11-5.

47. Jenkins DJA, Kendall CWC, Marchie A, et al. Dose response of almonds on coronary heart disease risk factors: blood lipids, oxidized low-density lipoproteins, lipoprotein(a), homocysteine, and pulmonary nitric oxide: a randomized, controlled, crossover trial. Circulation. 2002;106:1327-32.

48. Jenkins DJA, Kendall CWC, Marchie A, et al. Effects of a dietary portfolio of cholesterol-lowering foods vs lovastatin on serum lipids and C-Reactive protein. JAMA. 2003;290:502-10. 